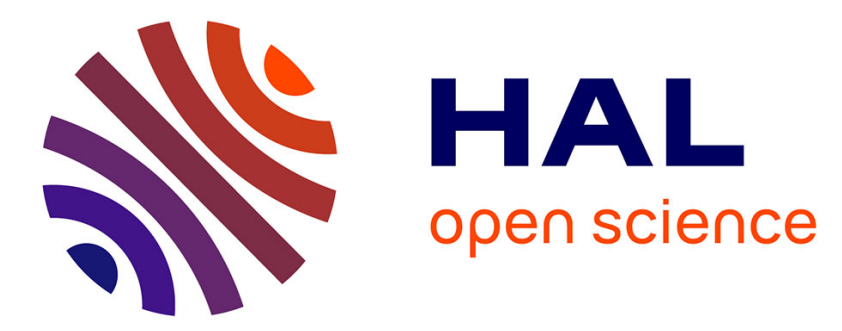

\title{
De l'observation du chercheur à l'accompagnement du dirigeant
}

\author{
Carole Drucker-Godard
}

\section{To cite this version:}

Carole Drucker-Godard. De l'observation du chercheur à l'accompagnement du dirigeant. @GRH, 2013, 6, pp.67-89. hal-01625218

\section{HAL Id: hal-01625218 \\ https://hal.parisnanterre.fr/hal-01625218}

Submitted on 21 Nov 2017

HAL is a multi-disciplinary open access archive for the deposit and dissemination of scientific research documents, whether they are published or not. The documents may come from teaching and research institutions in France or abroad, or from public or private research centers.
L'archive ouverte pluridisciplinaire HAL, est destinée au dépôt et à la diffusion de documents scientifiques de niveau recherche, publiés ou non, émanant des établissements d'enseignement et de recherche français ou étrangers, des laboratoires publics ou privés. 


\section{DE L'OBSERVATION DU CHERCHEUR A L'ACCOMPAGNEMENT DU DIRIGEANT}

Par Carole Drucker-Godard

\section{Introduction}

L'article s'inscrit dans la thématique générale de l'accompagnement des acteurs dans l'organisation. Il s'attache à comprendre comment, au fil de sa recherche et de sa rencontre avec l'autre, le chercheur devient un accompagnateur. Précisément, cet article propose une réflexion a posteriori du chercheur sur son travail. Pourquoi et comment les dirigeants observés ont-ils été amenés, peu à peu, au fil de la recherche, à considérer le chercheur comme un accompagnateur? Pour répondre à cette question et établir une analogie entre chercheur et accompagnateur, nous relisons l'histoire de la recherche à la lumière des travaux sur l'accompagnement et de nos données issues du terrain.

Depuis les années 1990, les pratiques d'accompagnement envahissent la plupart des secteurs professionnels, avec l'idée sous-jacente et récurrente de mener vers le progrès. Les formes d'accompagnement (coaching, counselling, mentoring, tutorat, conseil...) sont si diverses qu'il devient difficile d'en définir le terme.

Au départ de la recherche, tout se passe comme si l'offre d'un dirigeant rencontrait la demande d'un chercheur sans ajustement par la contractualisation, qui place la relation sous l'arbitrage de la loi et ritualise l'action. Ce constat est contradictoire avec la définition même de l'accompagnement et le sens offreur/demandeur est inversé. Pourtant, il semble que le binôme dirigeant /chercheur ait tout à fait sa place dans la démarche d'accompagnement, initiant peut-être même une nouvelle forme d'accompagnement?

Les arguments qui seront développés dans cet article tenteront de mettre en lumière l'affinité entre notre processus de recherche et une forme d'accompagnement qui se réfère au guidage. Le chercheur va en effet accompagner le dirigeant en montrant le chemin, en mettant en évidence ses comportements, il avertit le dirigeant de la manière dont il gère ses priorités. Nous apportons dans cet article un témoignage de chercheur sur le terrain, devenu au fil de la recherche un confident et une aide précieuse pour le dirigeant. Nous nous interrogeons sur les conditions qui font que l'accompagnement devient une démarche naturelle, surtout pas 
prescrite, alors qu'il s'agit d'un chercheur qui ne souhaite qu'interroger, observer ou suivre un dirigeant dans son quotidien.

Nous examinerons dans une première partie la littérature sur l'accompagnement. Une seconde partie présentera la posture réflexive du chercheur et sa finalité. Enfin, à la lumière de la littérature et de la relecture de notre recherche, nous montrerons dans une dernière partie comment le design d'une recherche, avec sa problématique et sa méthodologie de recherche, est devenu naturellement, au fil des jours et de la recherche, une forme d'accompagnement. Nous mettrons notamment en lumière la manière dont la pratique du chercheur le mène inconsciemment à une pratique d'accompagnement. Pour preuve : «le chercheur est mon guide » (parole de dirigeant).

\section{L'accompagnement : formes et pratiques}

Depuis les années 80, le terme Accompagnement envahit à peu près tous les domaines de la vie. Avant d'exposer l'accompagnement des individus et en particulier, dans une démarche ressources humaines, celui des entrepreneurs et dirigeants, retenons l'idée que celui-ci « concerne les situations dans lesquelles il y a un acteur principal que, d'une manière ou d'une autre, il s'agit de soutenir, de protéger, d'honorer, de servir, d'aider à atteindre son but » (Le Bouëdec, 2002 : 14). Plus exactement, l'accompagnement est « une pratique qui vise à apporter des solutions personnalisées à des individus désaffiliés, en quête de repères dans un univers complexe et mouvant, censés y être autonomes ou capables de le devenir, tout en étant soumis à des injonctions toujours plus pressantes en termes de performance, d'efficacité et d'excellence » (Paul, 2004 : 18). Alors qu'Ardoino (2000) propose de rendre compte de l'accompagnement dans huit domaines de la vie, nous ne nous concentrons ici que sur les formes d'accompagnement des cadres dirigeants.

\section{1 - Les pratiques d'accompagnement des cadres dirigeants}

Le domaine socio-économique a multiplié les formes d'accompagnement personnalisé des cadres dirigeants, telles que le coaching, le counselling, le consulting, le tutorat ou le mentoring. Ces pratiques diffèrent largement selon le praticien et selon la situation à laquelle elles s'appliquent. Qu'en est-il du cas des cadres dirigeants en entreprise?

\section{Le coaching}

L'accompagnement des managers est de plus en plus souvent appelé coaching, c'est pourquoi nous développons davantage cette forme particulière d'accompagnement. L'introduction du coaching en France remonte à une vingtaine d'années et se définit par les fédérations professionnelles et donc les praticiens comme «l'accompagnement d'une personne à partir de ses besoins professionnels pour le développement de son potentiel et de ses savoir-faire » (Société française de coaching, constituée en $1996^{1}$ ) ou « un processus de développement humain qui implique une relation structurée, des stratégies, des outils et des techniques adéquats pour promouvoir, de manière durable, des changements souhaités, au bénéfice du coaché et potentiellement d'autres acteurs de l'organisation » (Cox, Bachkirova et Clutterbuck, 2010 : 1).

\footnotetext{
${ }^{1}$ Cité dans Queuniet, $2001: 12-17$.
} 
Le coaching devient par ailleurs un champ d'études pour les sciences de gestion en 2005. Il est alors dénoncé par les chercheurs, notamment Rappin (2005) qui critique les savoirs mobilisés par les coaches. À ce sujet, Persson et al. (2011) établissent une revue de littérature qui montre que les chercheurs s'intéressent à la fois à la littérature en sociologie, psychologie, anthropologie, philosophie et enfin en gestion pour alimenter leurs recherches sur le coaching en entreprise. En analysant la littérature la plus significative se référant au coaching, Persson (2006) met à jour sept « familles référentielles de cette pratique d'accompagnement, telles notamment la vie en entreprise mais aussi le sport, la communication interpersonnelle, familles synthétisées et redéployées plus tard par Rapin (2006) qui s'intéresse au coaching des dirigeants. Queuniet (2001) insiste, lui, sur l'objectif de valorisation de l'individu et de sa performance alors que l'étude menée par Cloet (2006) auprès de praticiens atteste de la grande hétérogénéité des pratiques mêmes de coaching. L'auteur dresse en effet neuf dimensions des différentes conceptions du coaching. Il est ainsi difficile d'en donner une unique définition, ce sur quoi insiste également Fatien (2008) dans ses travaux sur les ambiguïtés du coaching. L'auteur parlera plus tard de malléabilité du coaching (Fatien et al., 2012). Persson et Bayad (2007 : 163) ont néanmoins tenté de construire une définition de la notion de coaching en se basant à la fois sur les définitions fournies par les groupements professionnels et celles issues des praticiens du coaching (environ 80 sur la période 19922006). Ainsi, «le coaching est une affaire de personne (...), qui se traduit par un accompagnement à vocation d'aide (...), se déroule en entreprise (...) et vise un objectif ».

Le coaching auprès des dirigeants, en particulier, a bien pour fonction de développer les compétences et le potentiel des hommes de l'organisation (Boltanski et Chiapello, 1999), c'est le moyen de "favoriser l'engagement individuel et collectif en entreprise 》 (Persson, 2009 : 1). Le coaching de dirigeants concerne des individus qui, dans le contexte de leur situation professionnelle, ont formulé une demande d'aide (Paul, 2002). Chavel (2007 : 217), dans son ouvrage sur le coaching du dirigeant, revient particulièrement sur ce thème, montrant notamment que cette pratique « instaure un triple rapport de sens entre le sujet, son monde et autrui ». Il insiste par ailleurs sur la personnalité du coach de dirigeants. Notons que le coaching est une démarche d'accompagnement particulièrement pertinente pour l'entrepreneur qui se situe souvent dans un contexte de changement. Sur ce sujet précis, l'étude de Audet et Couteret (2006) est intéressante puisque, en décrivant les spécificités du coaching entrepreneurial à l'aide d'une littérature pourtant très limitée sur ce concept, les auteurs, en plus de nous livrer un modèle de succès du coaching entrepreneurial, insistent sur la nécessité d'une relation interpersonnelle privilégiée entre coach et entrepreneur et conclue sur une opposition « teaching » / « learning » pour que «l'entrepreneur développe son habileté à prendre du recul et sa réflexion sur son expérience d'apprentissage » (143). Sur ce même thème, Persson et Bayad (2007) étudient l'accompagnement des porteurs de projets pour comprendre le coaching entrepreneurial. Outre l'importance de l'interaction entre les deux acteurs, les auteurs soulignent le rôle de ce type particulier de coaching pour "permettre un apprentissage différent par l'action (...) et aider le porteur de projet à se doter d'une représentation réflexive de son projet entrepreneurial ». (150)

\section{Le counselling}

Rogers $^{2}$ énonce dès 1942 la méthodologie du counselling comme des «consultations directes avec un individu en vue de l'aider à changer ses attitudes et son comportement ». Sur cette base et selon les termes employés par Rogers, Paul (2002) nous rappelle que le counselling est

\footnotetext{
${ }^{2}$ Cité dans Paul, M. (2002), 31.
} 
aujourd'hui considéré à la fois comme du conseil, de l'aide, de la guidance et de l'assistance dans un processus de développement. Ainsi, le counselling auprès de dirigeants est une pratique centrée sur la relation plus que sur un problème ; il s'agit d'une rencontre entre un accompagnateur et un dirigeant en demande d'aide.

\section{Le conseil}

L'accompagnateur, dans ce cas précis nommé le consultant, est généralement un expert à qui un individu expose une situation précise, sans attendre de sa part de décision, seul un avis. Le conseil consiste donc à « accompagner une personne » (Lhôtellier, 2001 : 191) en vue d'élaborer ensemble une conduite à tenir pour répondre à un problème. Dans le cas des cadres dirigeants, nous parlons du conseil individuel, celui qui permet à un dirigeant "d'intégrer une action dans son histoire et dans ses interactions sociales »(Paul, 2002 : 33). Aujourd'hui, le conseil va au-delà de la résolution d'un problème, il implique des changements de comportement, du raisonnement et de la relation.

\section{Le tutorat}

Le tutorat est une fonction de l'entreprise qui se justifie dans le cadre d'un objectif de formation professionnelle. Ainsi, il se définit à la fois comme "relation d'aide entre deux personnes pour l'acquisition des savoir-faire et l'intégration dans le travail » et comme "élément d'un dispositif visant à rendre le travail formateur et l'organisation intégratrice " (Paul, 2002 : 37). On retrouve finalement une relation entre accompagnateur-accompagné qui pourrait s'apparenter à la relation maître-apprenti dans le but de transmettre un métier, des compétences, des savoir-faire. Outre ce modèle de tutorat traditionnel, il est important de mentionner que le tuteur est devenu, dans une approche plus récente et plus moderne, un « facilitateur » (Vincent, 1982), dont le rôle est d'aider un individu (en général jeune) à capitaliser sur une expérience, à réfléchir à un vécu. On comprendra que dans cette perspective, le tutorat concerne moins les cadres dirigeants que les jeunes employés.

\section{Le mentoring}

Le mentoring est clairement une pratique d'accompagnement professionnel. Pour autant, en France, il " n'a jamais été étudié en tant qu'approche importante qui devrait avoir des effets sur la performance dans tous les domaines » (Totchilova et Lévêque, 2002) ${ }^{3}$. Le mentoring se décompose en plusieurs phases qui ont chacune un objectif particulier. La relation qui s'instaure en phase de commencement se prolonge par des changements induits dans les attitudes ou les comportements en phase de déroulement. Enfin, le dénouement du mentoring doit permettre à l'accompagné de se débrouiller seul. Il s'agit d'une relation de « solidarité intergénérationnelle » (Paul, 2002). Le mentoring est ainsi considéré comme une relation «profitable », le plus souvent entre un manager expérimenté et un manager moins expérimenté, qui peut diversement se décliner dans une organisation ou une profession (Damart et Pezet, 2008). Pour affiner la compréhension de cette pratique d'accompagnement, Persson et Ivanaj (2009) ont mené une grande étude d'investigation pour établir un état des savoirs sur le mentoring en ayant recours à une étude scientométrique. Ils montrent ainsi la grande complémentarité entre deux formes d'accompagnement : le coaching qui a la faveur des praticiens français pour sa méthodologie et le mentoring, "structuré par les notions de durée, de sens, de transmission, de filiation ». Enfin, comme pour le coaching, on insiste

\footnotetext{
${ }^{3}$ Cité dans Persson, S. et Ivanaj, S. (2009) : 98.
} 
fortement dans la pratique du mentoring, sur la richesse de la relation entre accompagnateur et accompagné. Kalbfleisch et Davies $(1993)^{4}$, affirment d'ailleurs que le «principal facteur-clé de succès repose sur les qualités de communication des deux parties ».

Ces cinq pratiques d'accompagnement individuel sont les plus usuelles en entreprise. Elles présentent des similarités dans le sens où elles sont toutes dirigées vers la transmission et plus spécifiquement la transmission de savoirs, de savoirs-être ou de savoirs-faire. Même si ces différentes pratiques sont une mise en relation et une stratégie où l'accompagnateur s'adapte à la situation, il est selon Paul (2002), impensable de réduire l'accompagnement à une forme unique. D'une part, les demandes d'aide sont très diverses, d'autre part, les rôles des accompagnateurs sont multiples et enfin, les logiques varient entre apprentissage, développement, formation ou résolution de problème. Quels sont donc les principaux registres de l'accompagnement qui nous intéressent?

\section{2 - Les registres de l'accompagnement}

Notons que chacune des pratiques présentées ci-dessus change selon les objectifs, le public ou le style de l'accompagnateur. "Accompagner, c'est aussi cheminer ensemble, l'un exerçant une fonction spécifique à l'égard de l'autre » (Paul, 2002 : 68). L'analyse sémantique du verbe accompagner permet ainsi de distinguer trois registres du mot qui se rapprochent du travail de chercheur que nous avons effectué : conduire, guider et escorter (Paul, 2004). Notons qu'il s'agit souvent de synonymes employés pour le terme accompagner alors que ces trois verbes, à signification différente, font finalement bien apparaître trois registres distincts de l'accompagnement.

\section{Conduire}

Conduire consiste à mener quelqu'un quelque part en étant à la tête du mouvement, ce qui sous-entend les idées de commander, diriger ou gouverner. Il s'agit d'inciter un acteur à être dans une situation particulière et à le faire progresser. Le terme reprend donc l'idée d'accompagnement, d'influence, et de direction pris dans le sens à la fois d'autorité et d'orientation. On retrouve sous-jacente la notion de fermeté qui nous ramène de manière étonnante aux idées précédemment développées de relation très proche entre accompagnateur et accompagné. L'accompagnateur va soit imposer une direction ou juste communiquer le sens du chemin. Ainsi, il faut nuancer, dans le fait de « conduire » le positionnement des deux acteurs de l'accompagnement.

\section{Guider}

Guider se rapporte au registre du conseil et de l'orientation. Il s'agit toujours d'accompagner quelqu'un en montrant le chemin mais avec l'idée de veiller à cette route. Dans ce sens, guider « aide à reconnaître le chemin, à choisir une direction» (Paul, $2002: 70)$. Se retrouvent ici les idées de voie et orientation à choisir, de chemin, mais aussi de délibération en vue d'une décision à prendre : «guider consiste à mettre devant les yeux (...) à faire reconnaître ». Ainsi, l'accompagnant éclaire et avertit.

\footnotetext{
${ }^{4}$ Cité dans Audet, M.J et Couteret, P. (2006) : 146.
} 


\section{Escorter}

Escorter consiste à accompagner pour guider, surveiller, défendre. Le terme reprend l'idée de réconfort, de prémunir contre un danger. La nuance par rapport à conduire et guider se situe au niveau de la protection apportée à autrui. Il en découle une relation nouvelle entre accompagnateur et accompagné puisque dans ce cas l'accompagné est en situation de faiblesse ou de défaillance ; la relation est ainsi déséquilibrée.

Le tableau 1 résume les trois registres de l'accompagnement :

Tableau 1 - Les registres de l'accompagnement (Paul, 2004 : 72 )

\begin{tabular}{|l|l|l|}
\hline \multicolumn{1}{|c|}{ Conduire } & \multicolumn{1}{|c|}{ Guider } & \multicolumn{1}{c|}{ Escorter } \\
\hline $\begin{array}{l}\text { Idées d'influence, incitation, } \\
\text { autorité pour mener avec soi } \\
\text { dans une certaine direction. }\end{array}$ & $\begin{array}{l}\text { Idées de délibération afin } \\
\text { d'éclairer l'orientation, la voie, } \\
\text { le chemin à choisir. }\end{array}$ & $\begin{array}{l}\text { Idées de projection, } \\
\text { de préparation ou de } \\
\text { recouvrement de dispositions. }\end{array}$ \\
\hline \multicolumn{2}{|c|}{ Accompagner, c'est participer d'une... } \\
\hline $\begin{array}{l}\text { Dynamique de progression, } \\
\text { d'élévation consistant à } \\
\text { se diriger dans la société } \\
\text { selon une certaine ligne de } \\
\text { conduite. }\end{array}$ & $\begin{array}{l}\text { Dynamique de développement } \\
\text { supposant connaissance de } \\
\text { soi (se situer) et projection de } \\
\text { soi (orientation). }\end{array}$ & $\begin{array}{l}\text { Dynamique de construction ou } \\
\text { de rétablissement, impliquant } \\
\text { étayage, réparation, } \\
\text { restauration ou mobilisation } \\
\text { de ressources individuelles. }\end{array}$ \\
\hline
\end{tabular}

Les pratiques et registres de l'accompagnement sont ainsi divers et nombreux. Cet état de l'art nous permettra dans la suite de l'article de nous situer, en tant que chercheur, et de voir de quelles pratiques et postures de l'accompagnement nous nous rapprochons le plus dans notre travail auprès de dirigeants. À cette fin, nous présentons dans une deuxième partie notre posture de chercheur.

\section{La démarche du chercheur: une démarche de réflexivité}

Pour comprendre la démarche de recherche qui conduit cet article, il faut revenir brièvement sur l'objet de la recherche initiale. La recherche porte au départ sur l'observation du quotidien de dix dirigeants d'organisations diverses. Il s'agit d'examiner la manière dont le dirigeant sélectionne les préoccupations décisionnelles qu'il va placer sur son agenda décisionnel et comment il les fait évoluer jour après jour sur cet agenda. Le travail empirique a consisté à suivre la dynamique de l'agenda du dirigeant pendant un mois, par le biais de questionnaires à remplir chaque soir par le dirigeant et d'un entretien hebdomadaire d'une durée de deux à trois heures. La méthodologie, basée sur des études de cas, est une méthodologie qualitative qui se situe dans un positionnement épistémologique interprétativiste.

Pour son travail réflexif sur cette recherche, l'auteur s'appuie en particulier sur deux matériaux précieux : son journal de bord et ses données issues du terrain. Explicitons auparavant l'intérêt de la posture réflexive pour la recherche en Sciences de Gestion. 


\section{1 - De la démarche réflexive et de son utilité}

Il existe une littérature abondante sur le cheminement réflexif en particulier en Sciences de l'Éducation et en Sociologie de l'action. Les définitions sont multiples et s'adaptent à divers contextes. La comparaison des différentes définitions permet de conceptualiser la démarche réflexive comme « une analyse de son expérience passée, présente, future et conditionnelle » (Paturel, 2009 : 8), une démarche de structuration et de transformation des perceptions et du savoir professionnel, qui vise l'émergence ou l'explicitation d'un savoir tacite, faisant appel à une prise de conscience de son cheminement professionnel. Dans son travail sur l'implication du praticien et du chercheur, Paturel (2009) décrit les quatre étapes de la réflexivité : intuition (impression, ressenti), explicitation (prise de recul par rapport à ses intuitions), description (contextualisation des faits, questionnement sur sa subjectivité) et enfin analyse (interprétation). C'est bien ce cheminement que nous suivrons en « relisant » nos travaux. D'après Schön (1994), une démarche réflexive se déclenche souvent suite au désir d'améliorer sa pratique. Il en est de même pour le chercheur : relire sa recherche permet de mettre en avant la portée et les limites du recueil des données, du design de recherche, du positionnement épistémologique et d'en tirer des leçons. Pour notre part, cette réflexion a posteriori sur notre démarche est plus une prise de conscience d'une part de la relation établie entre chercheur et objet de recherche (les dirigeants) et d'autre part de notre propre démarche qui s'apparente étonnamment à celle de l'accompagnement.

Nous défendons la thèse selon laquelle la manière dont le chercheur « fait son terrain » n'est pas anodine et tout chercheur devrait en amont s'interroger sur sa pratique (Whittington, 2006), notamment en questionnant ses pratiques de collecte des données et plus généralement du terrain, ce qui est en partie l'objet de cet article. Tout chercheur souhaitant aujourd'hui s'engager dans un travail qualitatif dispose, à travers articles et ouvrages, de descriptions méthodologiques techniques (design, collecte, analyse des données), lesquelles laissent souvent à penser qu'il est aisé de conduire une recherche qualitative. Or, tous les chercheurs qui ont vécu un processus de recherche qualitatif savent que la réalité d'une vie sur le terrain est loin d'être simple. C'est un exercice hautement délicat qui demande une attention, une imagination, une réactivité et une remise en cause de tous les instants. La recherche qualitative ne naît que par les efforts constants du chercheur qui doit véritablement «faire » son projet et son terrain. Ainsi, si la méthode est largement diffusée, la pratique qualitative est souvent passée sous silence (auteur et al., 2007). Ce constat est d'ailleurs repris par Solé (2007 : 286) : «lorsqu'il écrit son livre ou son article, le chercheur construit une histoire linéaire, logique, contrôlée, lisse. Cette histoire n'est pas celle qu'il a vécue » et «les livres de méthodologie et d'épistémologie ne parlent pas du chercheur au travail ». L'auteur s'élève même contre l'absence de formation au terrain des jeunes chercheurs. Il va jusqu'à dire que la formation à la recherche devrait passer par l'apprentissage «du sujet au terrain ». Solé (2007 : 304) insiste sur la nécessité de connaître le travail du chercheur autant que les résultats de sa recherche : «dans ses publications et communications, le chercheur ne présente pas son travail, il expose les résultats auxquels il est parvenu. La littérature de recherche contient une confusion majeure : l'on confond résultats obtenus et le processus de travail par lequel ils ont été produits ». Finalement, le chercheur, dans la restitution de ses travaux, suit des étapes logiques de démonstration, d'argumentation, de citation d'auteurs et de théories parce que c'est la coutume pour « convaincre ses pairs » mais le lecteur perd quelque chose de précieux sur le vécu du chercheur, sa démarche, ses émotions, son histoire du terrain. Relater précisément la démarche du chercheur serait ainsi un moyen, pour les chercheurs-apprentis, de limiter la confusion issue des ouvrages méthodologiques. Pour cela, un des moyens les 
plus efficaces est certainement de livrer ce que l'on nomme indifféremment journal de bord, récits de vie, ouvrages de souvenirs, correspondances avec des collègues, ou monographies...

Le travail réflexif trouverait donc toute son utilité pour l'apprentissage de la pratique du terrain.

\section{2 - Un regard sur le travail du chercheur a posteriori : la relecture du journal de bord et des données recueillies}

«Les témoignages des chercheurs sur leur travail sont rarissimes » (Solé, 2007 : 285). Au cours de notre étude longitudinale, nous avons tenu un journal de bord que nous n'avons jamais livré car considéré comme un outil personnel et intime, mais dont la lecture nous aide aujourd'hui à avoir un regard réflexif sur ce travail antérieur. Il nous semble que seuls les récits de vie sont à même d'offrir une vision de la réalité de la recherche et du terrain (Drucker-Godard et al., 2008). Le récit de vie, qui constitue une méthode à part entière, est une «stratégie d'accès au réel » dans une perspective exploratoire (Wacheux, 1996) ou une «forme d'entretien compréhensif » (Kaufmann, 2007). Joyeau et al. (2009) montrent d'ailleurs en quoi les récits de vie présentent un intérêt considérable dans le champ de la gestion des ressources humaines, à la fois pour le chercheur et pour le praticien. Notre étude empirique menée sur le terrain des dirigeants a ainsi fait l'objet d'une écriture en temps réel d'un journal de bord. Il s'agit d'un véritable témoignage avec ses anecdotes, ses difficultés et ses surprises. Les points clés de l'étude terrain y sont présentés, tout ce à quoi le chercheur a été confronté dans la réalité alors qu'il devait continuer à dérouler les fils de son enquête jusqu'à son terme et que son comportement était déterminant. C'est à la lecture a posteriori de ce journal de bord que nous prenons une posture réflexive qui nous permet de relater la recherche telle que nous l'avons faite et vécue et d'envisager ensuite un rapprochement inattendu avec les pratiques d'accompagnement. C'est par exemple dans ce journal ou à travers les entretiens que l'on peut relire certaines phrases des dirigeants : « si seulement je savais comment je gère mes priorités! J'en n'ai aucune idée mais j'aimerais que vous m'accompagniez dans cette démarche », ou "décidément vous devenez mon coach avec vos questions sur mes activités», ou encore «le chercheur est mon guide ». C'est aussi à travers ces récits de vie et ces données issues de nos entretiens que nous pouvons relire l'histoire de la recherche avec l'évolution des relations entre chercheur et dirigeant, par exemple : «quelles confidences je finis par vous faire » ou «votre étude se termine, au début je pensais pas pouvoir faire vos questionnaires mais là j'aimerais que vous continuiez à me suivre, j'ai appris plein de choses sur moi et ma façon de gérer mes priorités, on peut pas s'arrêter » ou enfin «je vous avoue maintenant que je pensais arrêter les questionnaires au bout de trois jours, mais quand vous venez le lundi et me posez des questions, je comprends plein de choses sur ma gestion du temps, $j$ 'en ai besoin maintenant, hum, un peu comme une drogue».

Que de phrases issues du journal de bord ou des entretiens qui, a posteriori, nous interpellent sur notre démarche : n'avons-nous pas fait, sans le savoir, de l'accompagnement? N'avonsnous pas été, au-delà d'un chercheur, un guide comme le déclare un dirigeant ? Et avonsnous, dans ce cas, suivi une démarche particulière d'accompagnement avec notre propre posture d'accompagnateur ? À la lumière de cette réflexion a posteriori de notre travail, nous tentons de répondre à ces questions. 


\section{Comparaison des démarches et pratiques du chercheur et de l'accompagnateur}

Au début de la recherche, les dirigeants ont répondu aux questionnaires parce qu'ils avaient accepté d'aider le chercheur mais peu à peu la relation s'est presque inversée. Les dirigeants commençaient en effet à être en attente de réponses semblant prendre un réel plaisir lors de nos entretiens. Aucun des dix dirigeants n'a annulé un des rendez-vous hebdomadaires et la plupart nous ont même accordé plus des deux heures initialement prévues. Le seul dirigeant peu convaincu de l'intérêt du sujet au début de la recherche nous a demandé de « continuer à l'accompagner ", selon ses propres termes, après l'étude. Il s'agissait d'un cheminement commun et à double sens. La conception même de l'accompagnement atteste que cet « accompagnement de la vie ordinaire » (selon l'expression de Fustier, 1993) pose au travers $\mathrm{du}$ «partage du quotidien » les fondements d'un « vivre-avec ». Comment, chercheur, avonsnous au fil du temps «endossé » un rôle d'accompagnateur et finalement évolué d'un statut à un autre?

\section{1 - La démarche : comment un design de recherche devient peu à peu une forme d'accompagnement}

C'est le plus généralement le dirigeant qui prescrit une aide à ses collaborateurs sous forme d'accompagnement, mais il n'est pas rare que le dirigeant ait lui-même besoin d'un accompagnateur, le plus souvent sous forme de coach, counsellor ou conseil.

Pour rapprocher la démarche du chercheur de celle de l'accompagnateur, nous montrons comment un design de recherche, avec sa problématique et sa méthodologie de recherche, peut s'assimiler à une démarche d'accompagnement, alors que le chercheur n'en avait au départ aucune prétention.

\subsection{1 - Une problématique de chercheur}

La recherche porte sur l'observation du quotidien de 10 dirigeants. Nous nous plaçons dans un contexte de recherche, c'est-à-dire qu'à l'inverse de la démarche traditionnelle d'accompagnement ce n'est pas le dirigeant qui fait appel à un accompagnateur mais un chercheur qui fait appel à un dirigeant pour comprendre la manière dont il gère chaque jour ses priorités. Nous sommes donc au départ dans un contexte de travail qui ne peut ni ne veut prétendre à un travail d'accompagnement. Il n'y a aucune demande, aucune prescription, aucune considération financière, aucun contrat car aucune obligation mutuelle qui définit les règles entre employeur et employé (Rousseau, 1989). D'ailleurs, Paul (2004) note que, parce qu'il est une présence institutionnalisée auprès d'autrui, l'accompagnement peut aboutir à des pratiques contradictoires selon qu'il s'agisse d'accompagner (coacher) des acteurs dans une visée de performance, accompagner (orienter, guider, conseiller) autrui dans les aléas transitionnels de son parcours, ou accompagner (soutenir, aider, protéger, réparer) un individu poussé à la marge par la précarité ou les revers existentiels. Aucun des dirigeants observés n'était dans une de ces situations et donc aucun n'était en demande d'aide à un moment de sa vie professionnelle.

Outre l'intérêt exprimé par les dirigeants pour la problématique de recherche, la relation qui s'est instaurée entre le dirigeant et le chercheur basée sur la confiance, la confidence et le 
respect mutuel est un point très important qui nous acheminera peu à peu, nous semble-t-il, vers une démarche d'accompagnement, tout comme la méthodologie de recherche, comme nous le voyons dans la section suivante.

\subsection{2 - Mais une méthodologie de recherche qui s’apparente à une démarche d'accompagnement \\ Le recueil des données}

On attribue communément au processus d'accompagnement trois phases essentielles (Lescarbeau, 2002) : l'initialisation, le déroulement et le dénouement. La première vise à l'établissement d'une relation formalisée par un contrat précisant les objectifs à atteindre et les moyens utilisés. La seconde, considérée comme accompagnement proprement dit, désigne le cheminement en commun pour la résolution du problème, tandis que la troisième phase se réfère aux opérations de synthèse. Il apparaît que nous avons en grande partie suivi ce processus d'accompagnement dans notre design de recherche.

En effet, nous avons en premier lieu expliqué très clairement l'objectif et l'intérêt de la recherche aux dirigeants lors d'un premier entretien. La relation n'a certes pas été formalisée officiellement par un contrat écrit mais il s'agissait d'une sorte de contrat moral ou psychologique (Rousseau, 1989), qu'aucun des dirigeants n'a d'ailleurs rompu pendant le mois d'étude. Les objectifs de la recherche et les moyens utilisés ont également été explicités aux secrétaires de direction. Concernant le déroulement de la recherche, nous avons expliqué très en détail toute la méthodologie mise en place. Il semble que cette première phase de la méthodologie de recherche s'apparente à la phase d'initialisation de l'accompagnement, décrite plus haut. L'utilisation des questionnaires ainsi qu'une série d'entretiens centrés correspondent, eux, à la phase de déroulement de l'accompagnement. La méthodologie de l'entretien centré repose sur un recueil d'informations qui fait référence à des situations concrètes dans lesquelles les personnes interrogées ont été impliquées. Nos entretiens ont en effet été relatifs aux données collectées dans les questionnaires (discussion); ils se référaient donc bien à des situations vécues par les dirigeants interrogés (réflexion). De la même manière, comme le rappelle Tourette-Turgis (1996 : 17), «les personnes qui formulent aujourd'hui une demande d'accompagnement ne sont plus les clients de Freud, (...) mais des personnes imbriquées dans des situations où il est difficile de séparer le problème individuel $d u$ contexte social ». Enfin, la mise en évidence de schémas types de gestion des priorités (qui ne seront pas développés ici) pourrait s'assimiler à la troisième phase du processus d'accompagnement : le dénouement.

Sans être non plus une source de preuve principale, notre présence sur le terrain (plusieurs heures par semaine, lors des entretiens) a parfois fourni des informations additionnelles intéressantes. La présence, rappelons-le, constitue une nécessité dans la démarche d'accompagnement.

Ainsi, nous proposons une analogie entre notre méthodologie de recueil et d'analyse des données et le processus d'accompagnement dans sa globalité.

\section{L'étude de cas}

Notre travail a initialement fait l'objet d'une étude multi-cas. Tout comme l'accompagnateur lorsqu'il débute son travail, nous avons opté pour une démarche caractéristique d'une 
construction théorique, où nous ignorons, non en totalité mais en très large partie, la teneur de nos découvertes. Comme le soulignent Miles et Hubermann (1994 : 434) «les études

qualitatives (...) permettent de repérer directement ou de manière longitudinale les processus locaux sous-jacents à une série d'événements ou d'états et montrer comment ceux-ci mènent à des comportements spécifiques ». Il apparaît que c'est aussi l'objectif de l'accompagnement que d'explorer les comportements pour proposer des solutions d'amélioration des performances. La position défendue par Van Maanen (1979 : 520) justifie également notre choix méthodologique dans son approche et nous semble aussi correspondre aux objectifs de tout accompagnement : «Le label de méthodes qualitatives (...) couvre un ensemble de techniques interprétatives qui cherchent à décrire, décoder, traduire et généralement percer le sens et non la fréquence de certains phénomènes survenant plus ou moins naturellement dans le monde social. Opérer sur un mode qualitatif, c'est traiter des symboles linguistiques et donc tenter de réduire la distance entre le signifié et le signifiant, entre la théorie et la donnée, entre le contexte et l'action. » Tout comme un accompagnateur se doit de prendre en compte les dimensions contextuelles du phénomène qu'il étudie, le chercheur étudie finement la relation entre le dirigeant et le contexte dans lequel il travaille. Ainsi, il nous semble que l'accompagnateur comme le chercheur se retrouvent dans les propos de Miles et Huberman (1991 : 22), lorsqu'ils précisent que « les données qualitatives permettent des descriptions et des explications riches de processus sociaux ancrés dans un contexte local ». Elles donnent en effet la possibilité au chercheur et à l'accompagnateur de fournir des explications fécondes, qui tiennent compte de la spécificité des personnes interrogées.

Dans notre travail initial, nous nous sommes rattachés ici à la conception de Yin (1989) qui fait de l'étude de cas une méthode appropriée pour saisir les caractéristiques complexes de phénomènes sociaux (Yin, 1989 : 14). L'étude de cas propose trois applications, applications que l'on retrouve, nous semble-t-il, dans les objectifs de l'accompagnement :

- elle peut expliquer les liens causaux qui existent dans la vie quotidienne et qui sont trop complexes à appréhender par expérimentation ou questionnaire,

- elle peut décrire le contexte de la vie quotidienne et un phénomène en particulier,

- elle peut explorer des situations qui ne sont pas claires.

Ainsi, nous définissons notre stratégie de recherche de la manière suivante : "C'est une approche empirique qui interroge un phénomène contemporain dans un contexte réel quand les frontières entre le phénomène et le contexte ne sont pas clairement évidentes et dans lequel de multiples sources de preuve sont utilisées »(Yin, 1989 : 23). Le parallèle avec la stratégie d'accompagnement est troublant lorsque Paul (2004 : 309) insiste sur l'accompagnement comme outil approprié pour «naviguer dans un environnement spatial et temporel placé sous le sceau de l'incertitude, pour conjoindre des données jusqu'alors considérées isolément, pour se mouvoir dans les contradictions».

Outre le design de recherche qui peut aisément s'assimiler à une démarche d'accompagnement, il semble que la pratique du chercheur s'apparente elle aussi à la pratique de l'accompagnateur. 


\section{2 - De la pratique du chercheur à la pratique de l'accompagnateur \\ 3.2.1 - Forme et posture d'accompagnement du chercheur}

Nous tentons ici d'avancer que par sa démarche de « chercheur-accompagnateur », le chercheur devient un guide.

\section{Le couselling comme forme d'accompagnement par le chercheur}

Si, comme vu précédemment, l'élément de base de la relation d'aide est l'entretien, l'essentiel du travail d'accompagnement se joue dans la capacité à permettre à l'accompagné d'entrer au contact avec lui-même, avec son mode intérieur et, pour cette capacité, l'attitude du counsellor est la plus efficiente (Tourette-Turgis, 1996 : 56). Dans notre cas, la pratique du chercheur, incitant le dirigeant à mener un travail d'introspection, ressemble fortement à cette forme d'accompagnement. En effet, dans notre recherche comme dans l'accompagnement, le rapport (chercheur/dirigeant ou accompagnateur/accompagné) s'est instauré au-delà du champ professionnel, dans la sphère même de l'existence humaine (Paul, 2004 : 30). Ce qui est au centre du processus, ce sont les personnes en situation et ce qui se passe entre elles, en relation, dans l'instant présent, et non le problème. Ce centrage sur la relation conduit certains praticiens à ne plus penser le counselling en termes de pratique d'orientation ou de psychothérapie mais bien en termes de rencontre (Condamin, 2000).

Si la relation est le lieu d'élaboration d'un questionnement permettant de « voir plus clair », elle prend nécessairement en compte la dimension temporelle comme temps de maturation nécessaire à chaque personne. Notons que le temps est un élément important de notre méthodologie de recherche. La reconstitution de l'évolution du portefeuille de préoccupations décisionnelles et la prise en compte du contexte impliquent l'observation de l'évolution du quotidien du dirigeant dans le temps. On retrouve a priori ici la relation d'accompagnement définie aussi comme temporaire. Finalement, il nous semble que l'étude processuelle, qui est la base de notre méthodologie de recherche est aussi la base de toute forme d'accompagnement. Ainsi, notre pratique de chercheur admet la subjectivité de l'acteur, le dirigeant, (tout comme celle du chercheur d'ailleurs) et offre l'opportunité d'une confrontation avec des réalités multiples car elle « expose plus directement la nature de la transaction » entre le chercheur et l'objet de recherche, et permet une meilleure évaluation de sa posture d'interaction avec le phénomène décrit (Lincoln et Guba, 1985 : 40).

«Comprendre » a impliqué dans notre recherche de tenter de retrouver les significations locales des dirigeants, c'est-à-dire des significations situées (dans l'espace) et datées (dans le temps), ce qui a été possible à travers les entretiens relatant les priorités quotidiennes des dirigeants. Ainsi, à l'instar de l'accompagnateur, nous avons été amenés à privilégier une démarche de recherche contextualisée pour analyser la gestion quotidienne du portefeuille de préoccupations décisionnelles du dirigeant.

Le concept-clé qui qualifie à la fois la rencontre comme possibilité et l'articulation relationtemps est celui de la présence. C'est à partir de cette base que se mettent en place l'écoute, le respect, l'empathie et la nécessaire référence aux connaissances qui permettent de repérer ce qu'il convient d'offrir à la personne non pas en terme de diagnostic à poser mais de temps à prendre pour concevoir un cadre et des outils appropriés (Condamin, 2000). À l'image de ce qui est évoqué dans les pages précédentes, notre pratique de chercheur se retrouve pleinement dans ces considérations. 
Comme l'indique Paul (2004 : 77), si le counselling s'inscrit dans la région sémantique de guider, c'est que le terme anglais provient du vieux français consel. Quant au verbe conseiller, il apparaît successivement avec le sens de guider quelqu'un dans sa conduite, puis indiquer quelque chose à quelqu'un. Il couvre, tout en les différenciant, les idées de conseiller et demander conseil. On voit apparaître initialement deux niveaux que l'on retrouve dans notre recherche : celui de la délibération intérieure et celui de la consultation ou de l'échange. Le counselling s'inscrit dans le champ sémantique de guider parce qu'il met l'accent sur la délibération intérieure suite à l'éveil, dans une démarche protectrice, attentive à l'individualité (Deschamps et al., 2000). Si l'on se réfère au tableau 1 (page 56), notre travail de chercheur se situe exactement dans ce registre de l'accompagnement : guider.

L'empathie qualifie précisément le processus relationnel et la structure de cette relation d'aide qui tend ici à devenir « un accompagnement d'une personne confrontée à une crise ou à des événements nécessitant d'opérer des changements dans le but de prendre ou de reprendre le contrôle sur sa vie et son histoire $»^{5}$. L'empathie, c'est justement notre posture de chercheur. Chaque dirigeant est unique, travaille dans un contexte unique, et possède une vision du monde qui lui est propre. Il nous a donc fallu essayer de partager le plus profondément possible leur vision des choses. Cela a supposé une certaine empathie avec le terrain, et donc de nous situer dans une perspective plutôt interprétativiste qui suppose de saisir la complexité du processus étudié en partageant les visions du monde des acteurs impliqués. Cela a été totalement et facilement accompli lors d'une étude exploratoire grâce à une période d'observation, puis les études de cas ont été le fruit d'une forte interdépendance entre les dirigeants et le chercheur, suite à une collaboration étroite et quotidienne. Nous cherchions avant tout à comprendre la dynamique du portefeuille de préoccupations décisionnelles des dirigeants au travers des interprétations qu'ils en font.

Tout comme le chercheur, l'accompagnateur (counsellor), centré sur les ressources de la personne, se place dans une posture empathique de dialogue et d'écoute, pour faciliter le développement individuel, en vue de trouver « un bon chemin ». Par cette posture empathique, le counsellor, au plus près des besoins personnels du client, peut faciliter une décision, ainsi éclairée par l'écoute et le dialogue.

\subsection{2 - Pistes sur les conditions du rapprochement chercheur - accompagnateur}

Au moins deux conditions apparaissent comme nécessaires pour tenter « d'assimiler » la recherche à un processus d'accompagnement.

D'une part, l'objet de la recherche. On a noté à plusieurs reprises l'intérêt des dirigeants pour notre problématique. Puisque le dirigeant n'est pas demandeur ni soumis à une prescription, il doit voir dans la problématique de recherche l'opportunité de trouver des réponses à des questions en suspens. Dans notre exemple, le chercheur est allé plus loin en obligeant le dirigeant à prendre du recul, à mener un véritable travail d'introspection. C'est cela que le dirigeant « redemande » au chercheur parce qu'il y trouve une source de réflexion, des explications.

\footnotetext{
${ }^{5}$ Association française de counseling.
} 
D'autre part, la relation chercheur / objet de recherche comme la relation accompagnateur/accompagné : «Ce qui fonde l'accompagnement tient à une obligation de contact et de proximité : toute la pratique professionnelle en relation à autrui s'en trouve transformée par la mise en ouvre d'une relation où les échanges sont visibles et valorisés comme créateurs de liens sociaux »(Paul, 2004 : 88). Ce sont ces mêmes proximités, relations et échanges que nous avons développés à travers notre méthodologie de recherche. Pour que cet échange se fasse entre les deux acteurs, outre l'empathie décrite plus haut, le chercheur doit prouver toute sa légitimité. L'idée que le dirigeant se fait du chercheur (ses compétences, son expertise, sa crédibilité, son désintérêt) l'aide probablement à créer une relation particulière qui s'apparente bien à une forme d'accompagnement. Le chercheur est à la fois crédible et légitime aux yeux du dirigeant. De par son statut, le chercheur intrigue et rassure à la fois. Il n'y a pas d'intimité, une juste distance sans implication ni influence du chercheur, mais une confiance mutuelle, une liberté dans les échanges qui a pu prendre une forme de confidence. Or, c'est cette attitude de l'accompagnateur (ou du chercheur) qui doit pousser le dirigeant à s'ouvrir pour se faire aider : il croit en les compétences de l'expert extérieur et prend plaisir à se connaître puis à se laisser guider. C'est donc dans la relation accompagnateur/accompagné que s'établit le véritable processus d'accompagnement, d'où l'importance du contrat moral entre les deux acteurs.

Enfin, il faut noter l'importance de la posture et de l'éthique du chercheur. Il convient, à la fois, de développer une réflexion sur un objet avec un statut d'extériorité et ne pas sousestimer l'implication personnelle du chercheur comme praticien de l'objet. Le chercheur se doit toujours de rester neutre, il n'est qu'un observateur qui cherche à décrire, décrypter, comprendre des comportements.

Si l'accompagnement s'inscrit dans une logique de compétence, portant sur l'individu, et soulignant l'implication personnelle et l'initiative individuelle, il insiste également sur la capacité de répondre aux situations difficiles, engageant la personne au-delà de son statut professionnel. Dès lors, la relation paradoxale que constitue compétence et accompagnement disparaît lorsqu'il s'agit du chercheur. La compétence relève du chercheur (et non plus du professionnel rémunéré) tandis que l'accompagnement se réfère, comme avant, au bénévolat. Ici, le chercheur a son rôle à jouer, plus que l'accompagnateur. Comme le souligne Paul (2004 : 118), on repère que toute relation d'accompagnement s'inscrit aujourd'hui dans un dispositif qui met en œuvre des politiques par la médiation d'organismes ou d'organisations au sein desquels l'accompagnement est d'abord une fonction : il s'acquitte d'une tâche particulière au regard de ces politiques et se décline en obligations pour le professionnel. Une des richesses du chercheur est probablement qu'il ne répond à aucune demande, aucune prescription mais suit un projet de recherche qui mobilise deux acteurs : lui-même et le dirigeant. La relation qui les lie est totalement désintéressée, ne donnera lieu à aucune rémunération qui serait préalablement définie dans un contrat et au sein d'un cadre institutionnel. La contractualisation s'opposant en quelque sorte à une appréhension empathique du chercheur, on peut dire que la relation qui se crée devient très riche, elle est plus souple et simplement humaine. Si le chercheur peut apporter des réponses au dirigeant, c'est notamment parce qu'aux yeux du dirigeant il est crédible et légitime. Il est vu non pas comme un professionnel, mais bien comme un acteur compétent, rigoureux, méthodique et en quête de réponses. Le statut, l'expérience et les compétences du chercheur restent pour le dirigeant des éléments forts. 


\section{3 - Discussion : vers une nouvelle forme d'accompagnement}

Par sa démarche, le chercheur devient un guide et « invente » peut-être même une nouvelle forme d'accompagnement.

\section{Guider et « refléter »}

Le chercheur a guidé le dirigeant, il a endossé tout naturellement et inconsciemment un nouveau rôle parce qu'en se livrant, les dirigeants lui ont donné ce rôle. En lui expliquant la manière dont il gère ses priorités au quotidien, le chercheur a proposé des recommandations aux dirigeants (déléguer, considérer différemment ses préoccupations personnelles et cachées, gérer différemment son temps...). Ce guidage a été reçu positivement dans la mesure où il a donné l'occasion à des dirigeants d'avoir un regard structuré sur leur agenda décisionnel et leur gestion du temps.

Les dirigeants ne prennent jamais de recul sur leur travail, pour des contraintes de temps évidentes. Ils ne se demandent jamais le soir ce qu'ils ont fait dans la journée, pourquoi, quel degré d'importance ils ont accordé à telle question, et pourquoi ils ont évacué tel sujet, par exemple. Ils ne se demandent pas si leurs priorités quotidiennes leur ont permis de faire avancer leurs objectifs pour l'organisation et/ou pour leur réalisation personnelle, ou si au contraire ils n'ont pas su se dégager des contraintes quotidiennes qui finalement ont freiné ces objectifs. Pour répondre à ces questions, les dirigeants ont besoin d'être aidés.

Nous insistons sur le fait que certains dirigeants nous ont demandé de reproduire à l'issue de la recherche l'étude à l'identique (avec les mêmes questionnaires et des entretiens hebdomadaires) pour les aider à regarder et à comprendre la manière dont ils gèrent leur agenda décisionnel et leurs priorités. À cet effet, nous avons fourni aux dirigeants une grille de lecture des gestions des priorités des dirigeants et une démarche méthodologique pour observer leur gestion des priorités.

Plus qu'une aide pour comprendre et agir, cette recherche a été un moyen pour chaque dirigeant de réaliser un travail d'introspection sur la façon d'organiser son agenda décisionnel et ses priorités, afin de prendre du recul et sans doute d'en améliorer la gestion, et peut-être aussi celle de son temps. C'est pour toutes ces raisons que nous proposons de formuler notre processus de recherche comme une nouvelle forme d'accompagnement qui consisterait non plus à conduire, guider et escorter l'accompagnant, mais à guider et « refléter ». En effet, par sa démarche, le chercheur aide le dirigeant mais surtout il lui offre le reflet fidèle de ses comportements, de la gestion de ses priorités et de son temps. Cette piste de recherche serait intéressante à approfondir dans un futur travail et il serait bien sûr utile de tester les hypothèses de rapprochement entre les démarches de recherche et d'accompagnement qui ressortent de cette réflexion a posteriori d'un chercheur qui relit son histoire.

\section{Conclusion}

L'examen de la littérature sur l'accompagnement couplé à une démarche réflexive qui nous a donné l'occasion de relire l'histoire d'une recherche à travers son journal de bord et ses entretiens nous a permis de vérifier les dires des dirigeants : le chercheur les a-t-il réellement 
accompagnés ? Pourquoi ont-ils changé leur perception pour confondre finalement le chercheur et l'accompagnateur?

La pratique du chercheur face à des dirigeants s'apparente finalement sur de nombreux points à une pratique d'accompagnement, celle du counselling, avec une posture de chercheur-guide. Même si l'ambition du chercheur est autre que celle de l'accompagnateur, et même si la relation avec l'objet de recherche est différente de la relation avec l'accompagné car non contractualisée et désintéressée, les similitudes sont nombreuses. On compte la méthodologie qui s'apparente aux trois phases du processus d'accompagnement, les objectifs de découverte, le besoin de situer la relation dans le temps et l'espace, la nécessité d'empathie et finalement une forme d'accompagnement. Tout se passe comme si en partant d'une étude de cas, le chercheur était devenu, malgré lui, un accompagnateur.

L'accompagnement tel qu'on le retrouve dans notre démarche de chercheur s'apparente à l'idée de délibération afin d'éclairer l'orientation, la voie, le chemin à choisir. Nous tentons en effet d'explorer les comportements des dirigeants pour comprendre et proposer des solutions d'amélioration de la gestion du quotidien et du temps. Le chercheur revendique également un travail d'accompagnement dans la mesure où il analyse en profondeur le cas d'un dirigeant, avec sa personnalité, ses comportements, ses décisions et actions pour expliquer, conseiller et guider.

Après avoir exposé quelques de pistes sur les conditions d'un rapprochement entre le travail de chercheur et d'accompagnateur, nous faisons l'hypothèse que le chercheur va au-delà de cet accompagnement car il ajoute à un des registres reconnus de l'accompagnement (guider) un nouveau registre : «refléter ». Ce constat pourrait ainsi militer pour une nouvelle forme d'accompagnement.

\section{Bibliographie}

- ARDOINOT, J. (2000). De l'accompagnement en tant que paradigme. Université Paris 8, Analyse de pratiques de formation, $\mathrm{n}^{\circ} 40$.

- AUDET, M.J. ET COUTERET, P. (2006). Le coaching comme mode d'accompagnement de l'entrepreneur. Revue internationale de psychosociologie, 12, 7, 141-160.

- BAUMARD, P. et al. (1999). La collecte des données et la gestion de leurs sources. In Thiétart, Méthodes de Recherche en Management, Dunod, Paris.

- BOLTANSKI, L., CHIAPELLO, E. (1999). Le nouvel esprit du capitalisme. Paris, Gallimard.

- BOUDON, R., BOURRICAUD, F. (1982). Dictionnaire critique de la Sociologie. Paris, PUF.

- LE BOUËDEC, G. (2002). La démarche d'accompagnement, un signe des temps. In Boutinet, J.P. et al., L'accompagnement dans tous ses états. Éducation permanente, 153, 1319.

- BOUTINET, J.P., PINEAU, G. (2002). L'accompagnement dans tous ses états. Education permanente, 153, 30-41.

- CHAVEL, T. (2006). Le coach de dirigeant, apogée et dépassement du leadership en entreprise. Thèse es Sciences de Gestion, Université Panthéon Assas. 
- CHAVEL, T. (2007) Le coaching du dirigeant. Éditions d'Organisation, Eyrolles, Paris, 196-225.

- CLOET, H. (2006). Le coaching : un OVNI ? Revue internationale de psychosociologie, $12,27,39-62$.

- CONDAMIN A. (2000). Réflexions sur la pratique du counselling dans une société en mutation. Université Laval.

- COX, E., BACHKIROVA, T. \& CLUTTERBUCK D. (eds.) (2009). The Complete Handbook of Coaching. Sage, London, New Delhi, Singapore, Washington DC, Los Angeles, 1.

- DAMART, S., PEZET, E. (2008). Étude exploratoire sur l'attitude des managers envers le coaching. Revue de Gestion des Ressources Humaines, 67, 38-48.

- DESCHAMPS, B., FATIEN, P \& GEINDRE, S. (2010). Accompagner le repreneur d'entreprise : conduire, escorter mais aussi guider. Gestion 2000, 3, 77-88.

- DRUCKER-GODARD, C., BOUTY I. (2007) The practice of researching strategy as practice: micro-level and multifaceted data collection process. 23th annual conference EGOS (European Group for Organizational Studies), Vienne, Autriche.

- DRUCKER-GODARD, C. (2008). Parlons terrain. Table ronde. $17^{e}$ congrès de l'AIMS (Association Internationale de Management Stratégique), participation et organisation en collaboration avec Bouty I., Garanto A. \& Musca G., Nice, France.

- FATIEN P. (2008). Des ambiguïtés de maux/mots du coaching. Nouvelle Revue de Psychosociologie, 6, Les ambiguités de la relation d'aide, 193-211.

- FATIEN, P. ET NIZET, J. (2012). Le coaching dans les organisations, Paris, Repères, La Découverte.

- FUSTIER, P. (1993). Les corridors du quotidien - La relation d'accompagnement dans les établissements spécialisés pour enfants. Presses Universitaires de Lyon.

- HOPPER, T., POWELL, A. (1985). Making sense of research into the organizational and social aspects of management accounting: a review of its underlying assumptions. Journal of Management Studies, 22, 5, 429-465.

- JOYEAU, A., ROBERT-DEMONTROND, P. \& SCHMIDT, C. (2009). Les récits de vie en Gestion des Ressources Humaines : principes, portée, limites. 20ème Congrès AGRH, Méthodes émergentes et recherche en GRH, Toulouse, 9-11 septembre.

- KALBFLEISCH, P., DAVIES, A.B. (1993). An interpersonal model for participation in mentoring relationships. Western Journal of Communication, 57, 4, 339-415.

- KAUFMANN, J.C. (2007). L'enquête et ses méthodes, l'entretien compréhensif. Paris, Armand Colin, $2^{\mathrm{e}}$ édition.

- KIMBERLY, J.R. (1976). Issues in the Design of longitudinal organizational research. Sociological Methods and Practical Issues, Newbury Park, Sage.

- LESCARBEAU, R. (2002). Une méthode d'intervention en développement organisationnel orientée vers l'individu : le coaching. Interactions, Québec, Université de Sherbrooke, 77-91.

- LHOTEllier, A. (2001). Tenir conseil. Délibérer pour agir. Paris, Seli Arsla.

- LINCOLN, Y.S., GUBA, E.G. (1985). Naturalistic inquiry. Newbury Park, Sage

Publications.

- MILES, M.B., HUBERMANN, A.M. (1991). Analyse des données qualitatives : recueil de nouvelles méthodes. Bruxelles, De Boeck Université.

- PATUREL, D. (2009). L'implication au cœur d'un processus de recherche. $20^{e}$ Congrès

AGRH, Méthodes émergentes et recherche en GRH, Toulouse, 9-11 septembre.

- PAUL, M. (2002). L'accompagnement : une nébuleuse. In Boutinet, J.P. et al.,

L'accompagnement dans tous ses états. Éducation permanente, 153, 43-55. 
- PAUL, M. (2004). L'accompagnement une posture professionnelle spécifique. Paris,

L'Harmattan.

- PERSSON, S. (2006). Les références du coaching, Analyse bibliométrique de la littérature francophone. Revue internationale de psychosociologie, 12, 27, 63-90.

- PERSSON, S. (2006). Le coaching, entre psychanalyse et problem solving. Revue internationale de psychosociologie, 12, 27, 13-20.

- PERSSON, S., BAYAD, M. (2007). L'accompagnement des porteurs de projet par le coaching entrepreneurial. Revue internationale de psychosociologie, 13, 31, 149-168.

- PERSSON, S., IVANAJ, S. (2009). Faut-il adopter le mentoring en France ? Etat des savoirs et perspectives généalogiques. Management \& Avenir, 25, 94-111.

- PERSSON, S. (2009). Mise en pespective des études empiriques sur le coaching : « des points de vue à la vue du point ». $20^{\text {ème }}$ Congrès AGRH, Méthodes émergentes et recherche en GRH, Toulouse, 9-11 septembre.

- PERSSON, S., RAPPIN, B. \& RICHEZ, Y. (2011). Les traverses du coaching. Dossier de la Revue internationale de psychosociologie, 42, 20-35.

- QUEUNIET, V. (2001). Le coaching acquiert sa légitimité, Entreprises et carrières, 597, 12-17.

- RAPPIN, B. (2005). Le réenchantement du coaching, Paris, Charles Antoni l'Originel.

- RAPPIN, B. (2006). L'accompagnement des dirigeants d'entreprise : vers une herméneutique du coaching. Thèse es Sciences de Gestion, Université Nice Sophia Antipolis. - ROBINSON, S.L., ROUSSEAU, D.M. (1994). Violating the psychological contract : not the exception but the norm. Journal of Organizational Behavior, 15, 3, 245-259.

- ROGERS, C. (1942). La relation d'aide et la psychothérapie. Paris, ESF.

- SCHÖN, D. (1994). Le praticien réflexif. À la recherche du savoir caché dans l'agir professionnel. Montréal, Éditions Logiques.

- SOLE, A. (2007). Le chercheur au travail. In Martinet A.C. (Dir), Sciences du Management, Epistémique, pragmatique et éthique, Paris, Vuibert, 285-30.

- TORCHILOVA-GALLOIS, E., LEVEQUE, M. (2002). La fonction du mentoring dans les structure éducatives, sportives et professionnelles. In Boutinet, J.P. et al., L'accompagnement dans tous ses états. Éducation permanente, 153, 155-165.

- TOURETTE-TURGIS, C. (1996). Le counselling. Paris, PUF.

- VAN MAANEN, J. (1979). Reclaiming qualitative methods for organizational research : a preface. Administrative Science Quaterly, 24, 539-550.

- WACHEUX, F. (1996). Méthodes qualitatives et recherche en gestion, Paris, Economica.

- WHITTINGTON, R. (2006). Completing the practice turn in strategy. Organization Studies, vol. 27, n5, 613-634.

- XHAUFLAIR, V., PICHAULT, F. (2009). La recherche-action au sein des partenariats inter-organisationnels : l'entrepreneur institutionnel peut-il théoriser son propre travail d'institutionnalisation? 20 Congrès AGRH, Méthodes émergentes et recherche en GRH, Toulouse, 9-11 septembre.

- YIN, R.K. (1989). Case study research : design and methods, Applied social research methods series. 5, Newbury Park, Sage Publications. 